\title{
Efficient Thermal Tuning Employing Metallic Microheater With Slow Light Effect
}

Yan, Siqi; Chen, Hao; Gao, Shengqian; Zhou, Feng; Xiao, Sanshui ; Ding, Yunhong; Dong, Jianji; Cai, Xinlun; Zhang, Xinliang

Published in:

I E E E Photonics Technology Letters

Link to article, DOI:

10.1109/LPT.2018.2837061

Publication date:

2018

Document Version

Peer reviewed version

Link back to DTU Orbit

Citation (APA):

Yan, S., Chen, H., Gao, S., Zhou, F., Xiao, S., Ding, Y., Dong, J., Cai, X., \& Zhang, X. (2018). Efficient Thermal Tuning Employing Metallic Microheater With Slow Light Effect. I E E E Photonics Technology Letters, 30(12), 1151-1154. https://doi.org/10.1109/LPT.2018.2837061

\section{General rights}

Copyright and moral rights for the publications made accessible in the public portal are retained by the authors and/or other copyright owners and it is a condition of accessing publications that users recognise and abide by the legal requirements associated with these rights.

- Users may download and print one copy of any publication from the public portal for the purpose of private study or research.

- You may not further distribute the material or use it for any profit-making activity or commercial gain

- You may freely distribute the URL identifying the publication in the public portal 


\title{
Efficient Thermal Tuning Employing Metallic Microheater With Slow Light Effect
}

\author{
Siqi Yan, Hao Chen, Shengqian Gao, Feng Zhou, Sanshui Xiao, Yunhong Ding, Jianji Dong*, Senior \\ Member, IEEE, Xinlun Cai ${ }^{*}$ and Xinliang Zhang, Senior Member, IEEE, Fellow, OSA
}

\begin{abstract}
Thermal tuning acts as one of the most fundamental roles in integrated silicon photonics since it can provide flexibility and reconfigurability. Low tuning power and fast tuning speed are long-term pursuing goals in terms of the performance of the thermal tuning. Here we propose and experimentally demonstrate an efficient thermal tuning scheme employing the metallic metal heater. The slow light effect in the photonic crystal waveguide is employed to enhance the performance of the metal microheater. Meanwhile, the metal microheater is integrated on the side of the waveguide rather than on the top. Thanks to both the slow light effect and the side-integrated microheater, the tuning efficiency is significantly enhanced and the response time as fast as $2 \mu \mathrm{s}$ is obtained. Since the thermal tuning with metal heater has been widely applied in silicon photonics, the proposed scheme may provide a valuable solution towards the performance enhancement of the thermal tuning in silicon photonics.
\end{abstract}

Index Terms-Integrated photonics, photonic crystal waveguides, silicon photonics.

\section{INTRODUCTION}

$I^{\prime}$ $n$ recent decades, we have witnessed the dramatic development of the silicon photonics towards the intense integration and multifunction because it holds several distinctive advantages including the compatibility with the mature complementary metal oxide semiconductor (CMOS) fabrication foundry as well as the cheap cost $[1,2]$. As one of the most feasible methods to manipulate the phase and intensity of the light on chip, thermal tuning has been extensively applied in integrated photonics including the large-scale photonic phased array antenna [3], integrated microwave photonics [4-6], optical artificial neural networks chip [7] and chip-scale tunable devices [8-10]. Up to now, the widely used structure to employ on-chip thermal tuning is to integrate the metal microheater on the top of the waveguide. By heating the

This work was supported under National Natural Science Foundation of China (61622502, and 61475052)( ${ }^{*}$ Corresponding author: Jianji Dong \& Xinlun Cai.)

Siqi Yan, Feng Zhou, Jianji Dong and Xinliang Zhang are with the Wuhan National Laboratory for Optoelectronics, Huazhong University of Science and Technology, Wuhan 430074 China. (e-mail: paulyan91@163.com, zhoufeng0803@qq.com,

xlzhang@mail.hust.edu.cn).

Shengqian Gao and Xinlun Cai are with State Key Laboratory of Optoelectronic Materials and Technologies, Sun Yat-sen University, Guangzhou 510275, China. (e-mail:lshandongs@qq.com, caixlun5@mail.sysu.edu.cn).

Sanshui Xiao and Yunhong Ding are with the Department of Photonics Engineering, Technical University of Denmark, DK-2800 Kongens Lyngby, Denmark. (e-mail: saxi@fotonik.dtu.dk, yudin@ fotonik.dtu.dk) waveguide using the microheater, the effective refractive index of the waveguide will be altered due to the thermo-optic effect, thus changing the phase of the light propagating through the waveguide. However, owing to the strong absorption of the light in the near-infrared wavelength induced by the metal, a thick silicon dioxide layer of $1 \sim 2 \mu \mathrm{m}$ is deposited between the heater and the waveguide to act as an isolation layer. As a result, the low thermal conductivity [11] of the silicon dioxide will impede the heat dissipation from the heater to the waveguides, thus leading to low tuning efficiency and slow response. Several schemes have been proposed to enhance the performance of the thermal tuning including free-standing waveguides [12], silicon waveguides with different doping levels [13] and the graphene microheater [14, 15]. Among these schemes, the free-standing waveguides have a slow response time of $170 \mu$ s while the different doped silicon waveguides have a relatively low tuning efficiency of $0.12 \mathrm{~nm} / \mathrm{mW}$ and complicated fabrication process. Although the slow light enhanced graphene microheater can simultaneously provide fast response time and high tuning efficiency, the graphene may induce excess loss to the devices and the fabrication process is complicated as it contains the wet-transfer process of graphene [15]. Therefore, a more feasible and economic solution is required to enhance the performance of the thermal tuning in silicon photonics.

In this paper, we present an efficient thermal tuning scheme employing the metal microheater and the slow light effect in photonic crystal waveguide $(\mathrm{PhCW})$. The metal microheater is fabricated on the side of the photonic crystal waveguide, rather than the top of the waveguide in previous schemes. This design can avoid the thick silica layer in conventional thermal tuning and enables a more efficient heat conduction from the heater to the waveguide. Besides, the structural parameters of the photonic crystal are optimized to obtain the slow light effect with large operation bandwidth and low loss, thus enhancing the tuning efficiency. Thanks to both side-integrated microheater and the slow light effect, an ultrafast average response time of $2.2 \mu \mathrm{s}$ is obtained. Besides, the power consumption required to reach per $2 \pi$ phase shift is $18 \mathrm{~mW}$ with the slow light enhanced tuning efficiency of $0.215 \mathrm{~nm} / \mathrm{mW}$. Since the thermal tuning with metal heater has been widely employed in silicon photonics, the proposed device may open up a novel way to achieve the effective tuning with low power consumption and fast response in large-scale integrated photonic chips. 


\section{Device STRUCTURE}
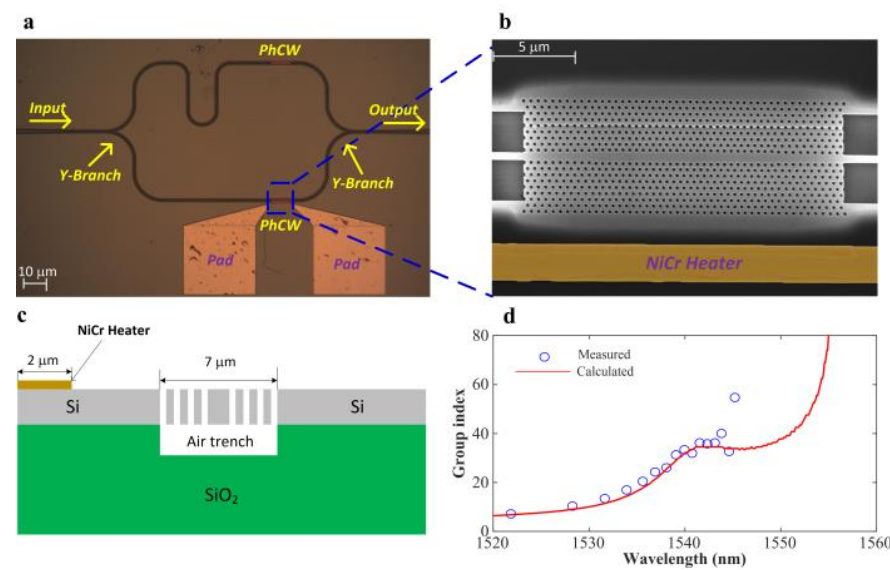

Fig. 1. (a)The microscopic image of the PhCW. (b) False-color Scanning Electron Microscopic (SEM) image of the thermal tuning part of the device. (c) The cross-section schematic of the thermal tuning part of the device. (d) The calculated and measured group index of the optimized $\mathrm{PhCW}$.

The microscopic image of the proposed thermal tuning structure is displayed in Fig. 1(a). To characterize the performance of the thermal tuning scheme, we designed and fabricated a Mach-Zehnder interferometer (MZI) on the commercial silicon-on-insulator (SOI) wafer with the top silicon layer of $220 \mathrm{~nm}$ thick and the buried oxide (BOX) layer of $2 \mu \mathrm{m}$ thick. Two grating couplers are employed to couple the light between the single mode fiber and the chip at the input and output of the MZI, respectively. The Y-branch acts as the optical power splitter into the upper and lower arms of the MZI. Both arms of the MZI consist single mode strip waveguide with the width of $500 \mathrm{~nm}$ and photonic crystal waveguide ( $\mathrm{PhCW}$ ), which are fabricated through using the electron beam lithography (EBL) and inductively coupled plasma (ICP) etching technologies. The length of the strip waveguide of the upper arm is designed to be $145.64 \mu \mathrm{m}$ longer than the lower arm in order to induce a fixed phase difference. Fig.1 (b) displays the zoom-in scanning electron microscopic (SEM) image of the active part of the PhCW-MZI. The BOX layer under the $\mathrm{PhCW}$ is selectively etched away by the buffered hydrofluoric acid (BHF) with the depth of $2 \mu \mathrm{m}$ and width of 7 $\mu \mathrm{m}$, as Fig. 1(c) shows in the cross-section view. The BOX layer under the strip waveguide remains unetched in order to ensure the mechanical stability of the structure. Afterwards, the $\mathrm{NiCr}$ microheater is formed by the standard ultraviolet (UV) lithography, metal deposition and lift-off process, successively. A $10 \mathrm{~nm} \mathrm{Al}_{2} \mathrm{O}_{3}$ layer is deposited on the wafer using atomic layer deposition between metal microheaters and the silicon in order to avoid the direct contact. The direct contact between the metal heater and silicon may lead to the formation of the Schottky junction, which may induce excess heating as well as the change of the free carrier density, thus affects the measured tuning efficiency and the performance of the device. Besides, the $\mathrm{Al}_{2} \mathrm{O}_{3}$ layer also contributes to the smoothness of the surface of the wafer. Since the optical field of the $\mathrm{PhCW}$ is mainly concentrated within the first three rows of the $\mathrm{PhCW}$ [16], the distance between the edge of the $\mathrm{NiCr}$ microheater and the center of the $\mathrm{PhCW}$ is set as $6.5 \mu \mathrm{m}$, which is enough to avoid the absorption loss. The width and length of the heater is $2 \mu \mathrm{m}$ and $10 \mu \mathrm{m}$, respectively. The lattice constant of the $\mathrm{PhCW}$ is $400 \mathrm{~nm}$ and the radius of the air holes of the $\mathrm{PhCW}$ is $101 \mathrm{~nm}$. In order to obtain slow light effect with large bandwidth and low loss in the $\mathrm{PhCW}$, the positions of the first and second rows have been optimized to move outward from their original position for $37 \mathrm{~nm}$ and $9 \mathrm{~nm}$ respectively. The group index of the $\mathrm{PhCW}$ is calculated using the 3D plane wave expansion (PWE) method [17] while the measured group index of the $\mathrm{PhCW}$ is obtained through the same method in our previous work [15]. The calculated and measured group index are shown in red solid line and blue circles in Fig. 1(d), respectively. One can find that the measured group index around 35 is achieved from $1540 \mathrm{~nm}$ to $1545 \mathrm{~nm}$, which matches well with our theoretical calculation. Within this wavelength range, the tuning efficiency could be enhanced according to our previous results.
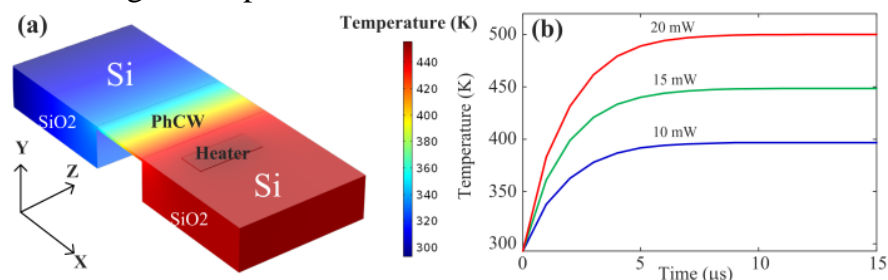

Fig. 2. (a)The thermal distribution of the proposed structure under the heating power of $10 \mathrm{~mW}$. (b) The dynamic responses of the proposed structure under different heating power of $10 \mathrm{~mW}, 15 \mathrm{~mW}$ and $20 \mathrm{~mW}$.

In order to investigate the thermal property of the proposed structure, the 3D finite element method (FEM) based on the Poisson's Equation is applied. In the theoretical analysis, the heating power of the microheater is set as $10 \mathrm{~mW}$. The thermal conductivities of the silicon, silica and $\mathrm{NiCr}$ heater are 148 $\mathrm{W} / \mathrm{m} \cdot \mathrm{K}, 1.38 \mathrm{~W} / \mathrm{m} \cdot \mathrm{K}$ and $17 \mathrm{~W} / \mathrm{m} \cdot \mathrm{K}$. The thermal conductivity of the $\mathrm{PhCW}$ is estimated to be $120.89 \mathrm{~W} / \mathrm{m} \cdot \mathrm{K}$ according to the air filling factor[18]. The calculated static thermal distribution is shown in Fig. 2(a). We can find that the heating power from the metallic heater can effectively increase the temperature of the $\mathrm{PhCW}$, thus inducing the change of the effective index of silicon. It should be noted that in the X-direction, although the temperature in the center of the $\mathrm{PhCW}$ is $40 \mathrm{~K}$ lower than the microheater, it is significantly higher than the conventional case with silica isolation layer and microheater on the top of the waveguide [19]. Meanwhile, there is a temperature difference across the $\mathrm{PhCW}$ in the $\mathrm{Y}$-direction. This phenomenon may not have a significant influence on the tuning efficiency since the optical field in the $\mathrm{PhCW}$ is limited in a relatively small area where the temperature distribution can be regarded as uniform. Besides, according to our previous work, the slow light effect can effectively lower the power consumption of the microheater[15]. Therefore, we can expect a higher tuning efficiency compared to the previous thermal tuning scheme. Besides, the dynamic response of the proposed thermal tuning scheme is also calculated. As we can see in Fig. 2(b), the 10\% 90\% switch times are around $2.5 \mu$ s for various heating power of $10 \mathrm{~mW}, 15 \mathrm{~mW}$ and $20 \mathrm{~mW}$, which is faster than most reported thermal tuning scheme with the metal microheater. 


\section{DEVICE CHARACTERIZATION}

We first measure the static response of the thermally tunable PhCW-MZI. The input light is emitted from a broadband laser source and adjusted by a polarization controller (PC) to reach maximum coupling efficiency before it is coupled into the chip by the grating coupler. At the output, the light is coupled into the SMF using another grating coupler and analyzed by an optical spectrum analyzer (OSA). The coupling loss of the grating coupler is $6 \mathrm{~dB}$ for each side.

As Fig. 3(a) shows, the transmission spectrum of the PhCW-MZI without external voltage is depicted in the blue solid curve. The total insertion loss of the device is $15 \mathrm{~dB}$ which includes the loss induced by the grating couplers, indicating the $3 \mathrm{~dB}$ insertion loss caused by the photonic crystal. We can find that the free spectral range (FSR)of the PhCW-MZI is measured to be $3.6 \mathrm{~nm}$. Moreover, three resonance dips are observed within the wavelength range from $1531 \mathrm{~nm}$ to 1541.3 $\mathrm{nm}$ and only the resonance dip at $1541.1 \mathrm{~nm}$ is located within the slow light region according to the result in Fig. 1(d). Next, external voltages are applied to the chip and the spectral responses are measured. The results are presented in Fig. 3(a). One can see that the interference dip at $1541.2 \mathrm{~nm}$ reaches the spectrum shift of one FSR (from the solid blue line to the solid green line) with a tuning power of $18 \mathrm{~mW}$, indicating a phase shift of $2 \pi$ of the light propagating through the PhCW. Meanwhile, the interference dip at $1541.2 \mathrm{~nm}$ experiences a larger shift than the dip at $1537.5 \mathrm{~nm}$ under the same heating power, thanks to the slow light effect. According to the spectrum shift, the measured resonance shift of the interference dips at $1537.5 \mathrm{~nm}$ (red line) and $1541.2 \mathrm{~nm}$ (blue line) as functions of the tuning power can be calculated and the results are given in Fig. 3(b).The tuning efficiencies of 0.215 $\mathrm{nm} \cdot \mathrm{mW}^{-1}$ and $0.132 \mathrm{~nm} \cdot \mathrm{mW}^{-1}$ are achieved with and without the slow light effect, respectively.
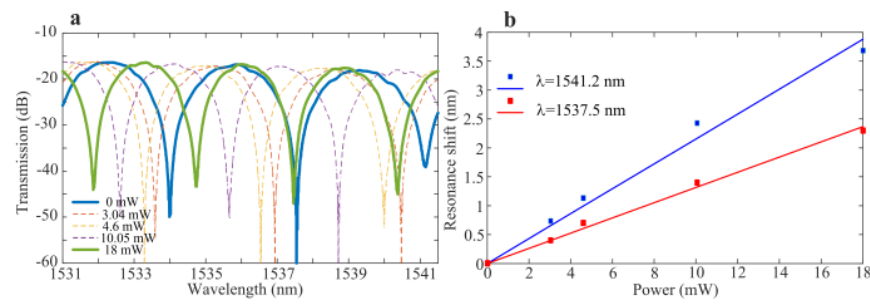

Fig.3. (a)The static spectral responses of the PhCW-MZI as functions of the heating power. (b) The resonance shift of the interference dips as functions of the heating power.

The dynamic property of the device is then tested by driving the chip with the electrical square waveform with the frequency of $40 \mathrm{kHz}$ and the power of $6.28 \mathrm{~mW}$, as the Fig. 4(a) displays. The wavelength of the input light emitting from a tunable laser source is fixed at $1539.2 \mathrm{~nm}$. At the output, the thermally modulated optical signal is received by a photodetector and recorded by an oscilloscope as the Fig. 4(b) shows. According to Fig. 4(b), the $10 \%$ to $90 \%$ rising and decaying times are measured to be $2.4 \mu \mathrm{s}$ and $2 \mu \mathrm{s}$, respectively. The measured response time fits well with the theoretical prediction in Fig. 2(b), which is the fastest response time compared to previous thermal tuning with metallic microheater in silicon photonics.
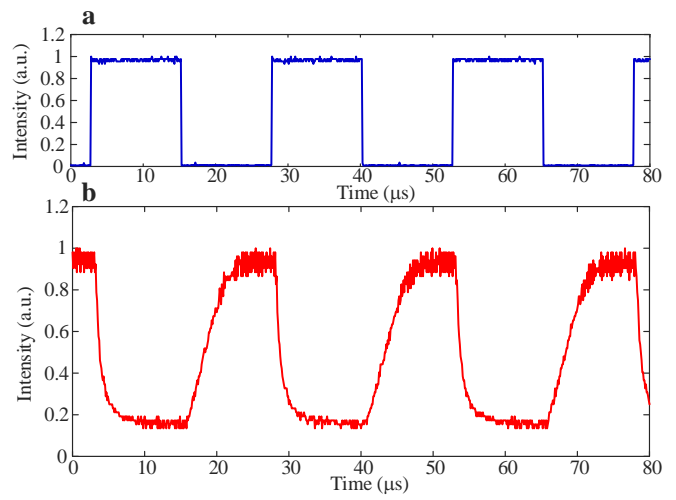

Fig.4. (a)The electrical driving signal with square waveform. (b) The corresponding output signal which is received by the photodetector.

\section{DISCUSSION}

To investigate the influence of the $\mathrm{PhCW}$ and the heater's length on the performance of the device, a longer $\mathrm{PhCW}$ with side-integrated microheater is fabricated and tested. As the Fig. 5(a) shows, the lengths of the $\mathrm{PhCW}$ and heater is increased to $20 \mu \mathrm{m}$ while keeping the other structural parameters unchanged. The tuning efficiencies with (blue dots and curve) and without (red dots and curve) the slow light effect are tested and the results are presented in Fig. 5(b). We can find that the tuning efficiency is lower compared to the case of the shorter length. The reasons may be attributed to the fact that the longer microheater has larger contact area with the silicon slab, thus resulting in more power dissipation in the direction opposite to the $\mathrm{PhCW}$. By applying $20 \mathrm{kHz}$ electrical signal with the square waveform to the chip, the 10\% 90\% response rise and fall time are measured to be $5 \mu \mathrm{s}$ and $6.5 \mu \mathrm{s}$, respectively. The slower response time in this case may also be due to the larger required power consumption to reach a $\pi$ phase shift.

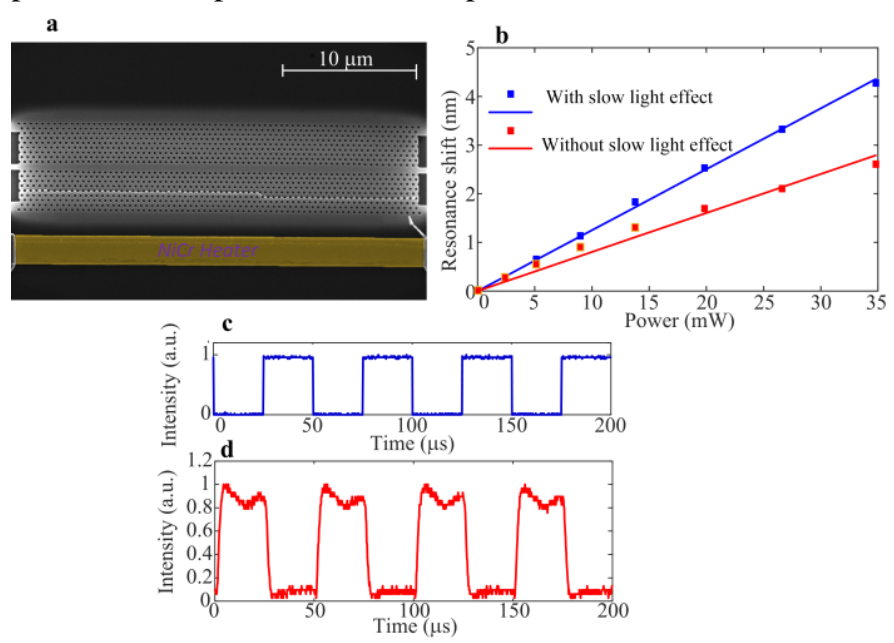

Fig.5. (a)The SEM image of the PhCW with side-integrated microheater of 20 $\mu \mathrm{m}$ (b) The measured tuning efficiency with (blue curve and dots) and without (red curve and dots).(c)The electrical driving signal with square waveform. (d) The corresponding output signal which is received by the photodetector.

The key parameters in our work are compared with previous work, which are shown in Table I. We can find that the power consumption can be significantly reduced by etching away the silicon substrate. But the response time is relatively high. 
Although graphene microheater holds the best performance parameters, the fabrication complexity is high since it requires the wet transfer process of graphene. Besides, the PhCW without the slow light effect has high power consumption and slow response time [20]. Here in our work, we combine the structural optimization of the microheater with the slow light to obtain low power consumption and fast response time simultaneously. It should be noted that the proposed microheater has a relatively narrow operation bandwidth of 10 $\mathrm{nm}$ compared to the standard thermal phase shifter. Broader operation bandwidth can be achieved at the expense of group index, indicating a lower tuning efficiency.

TABLE I

COMPARISON WITH PREVIOUS WORK

\begin{tabular}{cccc}
\hline \hline Structure & $\begin{array}{c}\text { Power } \\
\text { consumptio } \\
\text { n per } 2 \pi \\
(\mathrm{mW})\end{array}$ & $\begin{array}{c}\text { Response } \\
\text { time }(\mu \mathrm{s})\end{array}$ & $\begin{array}{c}\text { Fabrication } \\
\text { complexity }\end{array}$ \\
\hline $\begin{array}{c}\text { Silicon substrate etched away } \\
{[12]}\end{array}$ & 2.4 & 170 & Low \\
$\begin{array}{c}\text { Slow light enhanced graphene } \\
\text { microheater [15] }\end{array}$ & 3.99 & 0.55 & High \\
$\begin{array}{c}\text { PhCW without slow light [20] } \\
\text { PhCW with slow light (This } \\
\text { work) }\end{array}$ & 156 & 15.5 & Low \\
\hline \hline
\end{tabular}

\section{CONCLUSION}

In summary, we propose and experimentally demonstrate a novel thermal tuning structure with metal microheater. Thanks to the side-integrated microheater, a response time as fast as 2 $\mu$ s is obtained. The power consumption per $2 \pi$ is $18 \mathrm{~mW}$ with the slow light enhanced efficiency of $0.215 \mathrm{~nm} / \mathrm{mW}$. Besides, a longer $\mathrm{PhCW}$ with side-integrated microheater is also fabricated and investigated, which has lower tuning efficiency and slower response time. It should be noted that although $\mathrm{T}$. Baba et al. reported a similar idea to control PhCW with thermal tuning, they mainly focused on its application of the dispersion compensation [18]. However, in our study, we treat the side-integrated structure as a novel thermal tuning scheme to investigate its basic performance parameters including the fast response time and the slow light enhanced tuning efficiency. The proposed thermal tuning structures holds the advantages of ultrafast response time and slow light enhanced tuning efficiency, which has great potential in the application of large-scale reconfigurable photonic integrated circuits and integrated microwave systems.

\section{REFERENCES} John Wiley \& Sons, 2004. B. Jalali, and S. Fathpour, "Silicon photonics," Journal of Lightwave Technology, vol. 24, no. 12, pp. 4600-4615, 2006.

J. Sun, E. Timurdogan, A. Yaacobi, E. S. Hosseini, and M. R. Watts, "Large-scale nanophotonic phased array," Nature, vol. 493, pp. 195, 01/09/online, 2013.

J. S. Fandiño, P. Muñoz, D. Doménech, and J. Capmany, "A monolithic integrated photonic microwave filter," Nature Photonics, vol. 11, pp. 124, 12/05/online, 2016.

J. Capmany, I. Gasulla, and D. Pérez, "The programmable processor," Nature Photonics, vol. 10, pp. 6, 12/24/online, 2015. worlds," Nature Photonics, vol. 1, no. 6, pp. 319-330, 2007.

Y. Shen, N. C. Harris, S. Skirlo, M. Prabhu, T. Baehr-Jones, M. Hochberg, X. Sun, S. Zhao, H. Larochelle, D. Englund, and M. Soljačić, "Deep learning with coherent nanophotonic circuits," Nature Photonics, vol. 11, pp. 441, 06/12/online, 2017.

S. Gan, C. Cheng, Y. Zhan, B. Huang, X. Gan, S. Li, S. Lin, X. Li, J. Zhao, and H. Chen, "A highly efficient thermo-optic microring modulator assisted by graphene," Nanoscale, vol. 7, no. 47, pp. 20249-20255, 2015.

M. Liu, Y. Zhao, X. Wang, X. Zhang, S. Gao, J. Dong, and X. Cai, "Widely tunable fractional-order photonic differentiator using a Mach-Zenhder interferometer coupled microring resonator," Optics Express, vol. 25, no. 26, pp. 33305-33314, 2017/12/25, 2017.

S. Liao, Y. Ding, C. Peucheret, T. Yang, J. Dong, and X. Zhang, "Integrated programmable photonic filter on the silicon-on-insulator platform," Optics Express, vol. 22, no. 26, pp. 31993-31998, 2014/12/29, 2014.

T. Yamane, N. Nagai, S.-i. Katayama, and M. Todoki, "Measurement of thermal conductivity of silicon dioxide thin films using a $3 \omega$ method," Journal of Applied Physics, vol. 91, no. 12, pp. 9772-9776, 2002.

P. Dong, W. Qian, H. Liang, R. Shafiiha, D. Feng, G. Li, J. E. Cunningham, A. V. Krishnamoorthy, and M. Asghari, "Thermally tunable silicon racetrack resonators with ultralow tuning power," Optics Express, vol. 18, no. 19, pp. 20298-20304, 2010.

X. Li, H. Xu, X. Xiao, Z. Li, Y. Yu, and J. Yu, "Fast and efficient silicon thermo-optic switching based on reverse breakdown of pn junction,” Optics Letters, vol. 39, no. 4, pp. 751-753, 2014.

L. Yu, Y. Yin, Y. Shi, D. Dai, and S. He, "Thermally tunable silicon photonic microdisk resonator with transparent graphene nanoheaters," Optica, vol. 3, no. 2, pp. 159-166, 2016/02/20, 2016. S. Yan, X. Zhu, L. H. Frandsen, S. Xiao, N. A. Mortensen, J. Dong, and Y. Ding, "Slow-light-enhanced energy efficiency for graphene microheaters on silicon photonic crystal waveguides," Nature Communications, vol. 8, pp. 14411, 2017

J. Li, T. P. White, L. O'Faolain, A. Gomez-Iglesias, and T. F. . crystal waveguides," Optics Express, vol. 16, no. 9, pp. 6227-6232, 2008.

S. G. Johnson, and J. D. Joannopoulos, "Block-iterative frequency-domain methods for Maxwell's equations in a planewave basis," Optics Express, vol. 8, no. 3, pp. 173-190, 2001.

N. Ishikura, R. Hosoi, R. Hayakawa, T. Tamanuki, M. Shinkawa, and T. Baba, "Photonic crystal tunable slow light device integrated with multi-heaters," Applied Physics Letters, vol. 100, no. 22, pp. 221110, 2012

P. Dong, W. Qian, H. Liang, R. Shafiiha, N.-N. Feng, D. Feng, X. Zheng, A. V. Krishnamoorthy, and M. Asghari, "Low power and compact reconfigurable multiplexing devices based on silicon microring resonators," Optics Express, vol. 18, no. 10, pp. 9852-9858, 2010.

L. Gu, W. Jiang, X. Chen, and R. T. Chen, "Thermooptically Tuned Photonic Crystal Waveguide Silicon-on-Insulator Mach-Zehnder Interferometers," IEEE Photonics Technology Letters, vol. 19, no. 5, pp. 342-344, 2007. 